IJMMS 28:11 (2001) 637-652

PII. S0161171201011760

http://ijmms.hindawi.com

(C) Hindawi Publishing Corp.

\title{
SOLVABILITY OF KOLMOGOROV-FOKKER-PLANCK EQUATIONS FOR VECTOR JUMP PROCESSES AND OCCUPATION TIME ON HYPERSURFACES
}

\author{
N. G. DOKUCHAEV \\ (Received 31 January 2001 and in revised form 3 July 2001)
}

\begin{abstract}
We study occupation time on hypersurface for Markov $n$-dimensional jump processes. Solvability and uniqueness of integro-differential Kolmogorov-Fokker-Planck with generalized functions in coefficients are investigated. Then these results are used to show that the occupation time on hypersurfaces does exist for the jump processes as a limit in variance for a wide class of piecewise smooth hypersurfaces, including some fractal type and moving surfaces. An analog of the Meyer-Tanaka formula is presented.
\end{abstract}

2001 Mathematics Subject Classification. 60J55, 60J60, 60J75, 45K05.

1. Introduction. The local time or the occupation time of stochastic processes have been studied by many authors (cf. bibliography in survey papers [2, 5]). For example, the local time has been studied for the scalar Brownian motion and scalar semimartingales (cf. [9, 11]), for general one-dimensional diffusions (see [13]), for stable processes (see [10]).

For vector continuous processes, the distribution of the occupation time is also well studied. McGill [8] derived an analog of Tanaka formula for a solution of a scalar homogeneous nonlinear diffusion equation. Bass [1] investigated occupation time of multidimensional non-Markovian continuous semimartingales of general type and proved the existence of local time. Rosen and Yor [12] considered the occupation time for processes in the plain at points of intersections. Dokuchaev [3] studied occupation time for degenerating diffusion vector processes.

It appears that many important properties of Brownian local time do not hold for a case the occupation time of $n$-dimensional diffusion processes on $(n-1)$-dimensional hypersurfaces. For example, this occupation time cannot be presented as occupational measure in a case $n>1$. Hence it is not a perfect analog of Brownian local time.

The paper studies occupation time on hypersurface for Markov $n$-dimensional jump processes. As is known, analogs of Kolmogorov-Fokker-Planck equations for the probability distribution for jump processes are second-order integro-differential equations (cf. [4]). We extend solvability and uniqueness results for these equations for a case when there are generalized functions in coefficients (Sections 3 and 4). Then we show that the occupation time exists as a limit in variance, and an analog of the MeyerTanaka formula is derived, that is, the occupation time is presented as a stochastic integral (Section 6). Equations for the characteristic function of the occupation time are derived in Section 7. 
2. Definitions. Consider a standard probability space $(\Omega, \mathscr{F}, \mathbf{P})$, a standard $n$ dimensional Wiener process with independent components such that $w(0)=0$, and a Poisson measure $v(\cdot, t)$ in $\mathbb{R}^{m}$ such that the process $w(\cdot)$ and the measure $v(\cdot, t)$ are independent of each other. We assume that there exists a measure $\Pi(\cdot)$ in $\mathbb{R}^{m}$ such that $\mathbf{E} v(A, t)=t \Pi(A)$ for each measurable set $A \subset \mathbb{R}^{m}$.

Set $\tilde{v}(A, t) \triangleq v(A, t)-t \Pi(A)$.

Let $a$ be a random real $n$-dimensional vector such that $a$ does not depend on $w(\cdot)$ and $v(\cdot)$. We assume also that $\mathbf{E}|a|^{2}<+\infty$.

We consider an $n$-dimensional stochastic differential equation

$$
\begin{gathered}
d y(t)=f(y(t), t) d t+\beta(y(t), t) d w(t)+\int_{\mathbb{R}^{m}} \theta(y(t), u, t) \tilde{v}(d u, d t), \\
y(0)=a .
\end{gathered}
$$

The functions $f(x, t): \mathbb{R}^{n} \times \mathbb{R} \rightarrow \mathbb{R}^{n}, \beta(x, t): \mathbb{R}^{n} \times \mathbb{R} \rightarrow \mathbb{R}^{n \times n}$, and $\theta(x, u, t): \mathbb{R}^{n} \times$ $\mathbb{R}^{m} \times \mathbb{R} \rightarrow \mathbb{R}^{n \times n}$ are bounded and Borel measurable. We assume that the function $\theta(x, u, t)$ is continuous in $u$, the derivatives $\partial f(x, t) / \partial x, \partial \beta(x, t) / \partial x, \theta(x, u, t) / \partial x$ are bounded, and there exists a number $\delta>0$ such that $b(x, t)=(1 / 2) \beta(x, t) \beta(x, t)^{\top} \geq$ $\delta I_{n}>0$ (for all $x, t$ ), where $I_{n}$ is the unit matrix in $\mathbb{R}^{n \times n}$. Also, we assume that $\Pi\left(\mathbb{R}^{m}\right)<$ $+\infty$.

Under these assumptions, (2.1) has the unique strong solution (cf. [6, Theorem 2, page 242]).

Let a bounded hypersurface $\Gamma(t)$ of dimension $n-1$ be given for a.a. $t \in[0, T]$, and let $\partial \Gamma(t)$ be its edge (it can happen that $\partial \Gamma(t)=\varnothing$ ). Let some number $T>0$ be given. Let Ind denote the indicator function, and let $|\cdot|$ denote the Euclidean norm.

We will study the occupation time of $y(t)$ in $\Gamma(t)$. More precisely, we will study the limit of the random variables

$$
l_{\varepsilon}(T) \triangleq \frac{1}{\varepsilon} \int_{0}^{T} \operatorname{Ind}\{y(t) \in \Gamma(\varepsilon, t)\} d t
$$

as $\varepsilon \rightarrow 0+$, where

$$
\Gamma(\varepsilon, t) \triangleq\left\{x \in \mathbb{R}^{n}: \inf _{y \in \Gamma(t)}|x-y|<\frac{\varepsilon}{2}\right\} .
$$

SPACES AND CLASSES OF FUNCTIONS. Below $\|\cdot\|_{X}$ denotes a norm in a space $X$, and $(\cdot, \cdot)_{X}$ denotes the scalar product in a Hilbert space $X$.

Introduce some spaces of (complex-valued) functions. Let $H^{0} \triangleq L_{2}\left(\mathbb{R}^{n}\right), H^{1} \triangleq$ $W_{2}^{1}\left(\mathbb{R}^{n}\right)$, where $W_{q}^{m}\left(\mathbb{R}^{n}\right)$ is the Sobolev space of functions which belong to $L_{q}\left(\mathbb{R}^{n}\right)$ together with first $m$ derivatives, $q \geq 1$.

Let $H^{-1}$ be the dual space to $H^{1}$, with the norm $\|\cdot\|_{H^{-1}}$ such that $\|u\|_{H^{-1}}$, for $u \in H^{0}$, is the supremum of $(u, v)_{H^{0}}$ over all $v \in H^{0}$ such that $\|v\|_{H^{1}} \leq 1$. Let $\ell_{m}$ denote the Lebesgue measure in $\mathbb{R}^{m}$, and let $\overline{\mathscr{P}}_{m}$ be the $\sigma$-algebra of the Lebesgue sets in $\mathbb{R}^{m}$. We introduce the following spaces:

$$
C^{0} \triangleq C\left([0, T] ; H^{0}\right), \quad X^{k} \triangleq L^{2}\left([0, T], \overline{\mathscr{B}}_{1}, \ell_{1} ; H^{k}\right), \quad k=0, \pm 1,
$$

and $Y^{1} \triangleq X^{1} \cap C^{0}$, with the norm $\|u\|_{Y^{1}} \triangleq\|u\|_{X^{1}}+\|u\|_{C^{0}}$. 
The scalar product $(u, v)_{H^{0}}$ is assumed to be well defined for $u \in H^{-1}$ and $v \in H^{1}$ as well (extending it in a natural manner from $u \in H^{0}$ and $v \in H^{1}$ ).

Let $\mu$ be a real number such that

$$
\mu \in \begin{cases}(1,2) & \text { if } n=1, \\ \left(1, n(n-1)^{-1}\right) & \text { if } n>1 .\end{cases}
$$

We introduce the space $\mathcal{W}=W_{\mu}^{1}\left(\mathbb{R}^{n}\right)$ with the norm

$$
\|u\|_{\mathscr{W}} \triangleq\|u\|_{L_{\mu}\left(\mathbb{R}^{n}\right)}+\sum_{i=1}^{n}\left\|\frac{\partial u}{\partial x_{i}}\right\|_{L_{\mu}\left(\mathbb{R}^{n}\right)}
$$

and its conjugate space $\mathscr{W}^{*}$, as well as the space $\mathscr{X} \triangleq L^{\infty}\left([0, T], \overline{\mathscr{P}}_{1}, \ell_{1} ; \mathscr{W}^{*}\right)$.

3. On solvability of integro-differential equations. In this section, an integrodifferential analog of the Kolmogorov-Fokker-Planck equation is studied. Existence results theorems for these equations can be found in Carrany and Menaldy [4]. However, we will need to extend the existence results for a case when there are generalized functions in coefficients.

Let $\mathscr{A}=\mathscr{A}(t)$ be the parabolic operator generated by the process $y(t)$,

$$
\mathscr{A} \triangleq \mathscr{A}_{c}+\mathscr{g}
$$

where

$$
\begin{gathered}
\mathscr{A}_{c} v=\mathscr{A}_{\mathcal{C}}(t) v \triangleq \sum_{i, j=1}^{n} b_{i j}(x, t) \frac{\partial^{2} v}{\partial x_{i} \partial x_{j}}(x)+\sum_{i=1}^{n} \hat{f}_{i}(x, t) \frac{\partial v}{\partial x_{i}}(x), \\
\mathscr{g} v \triangleq \mathscr{F}^{\prime}(t) v-\Pi\left(\mathbb{R}^{m}\right) v, \quad \mathscr{F}^{\prime}(t) v \triangleq \int_{\mathbb{R}^{m}} v(x+\theta(x, u, t)) \Pi(d u),
\end{gathered}
$$

and where

$$
\hat{f}(x, t) \triangleq f(x, t)-\int_{\mathbb{R}^{m}} \theta(x, u, t) \Pi(d u) .
$$

Here $b_{i j}, \hat{f}_{i}, x_{i}$ are the components of the matrix $b$ and the vectors $\hat{f}, x$.

Let $Q \triangleq \mathbb{R}^{n} \times[0, T]$. Consider a boundary value problem in $Q$

$$
\frac{\partial V}{\partial t}+\mathscr{A} V+g V=-\varphi, \quad V(x, T)=R(x)
$$

As is known, problem (3.5) is uniquely solvable in the class $Y^{1}$ for $\varphi \in X^{-1}, R \in H^{0}$ and $g \in L_{\infty}(Q)$ (cf. [7]).

Condition 3.1. There exists a constant $c_{\pi}>0$ such that

$$
\mathbf{P}(\eta \in B) \leq c_{\pi} \ell_{n}(B) \ell_{n}(D)^{-1}
$$

for any $t \in[0, T]$, any bounded measurable set $D \subset \mathbb{R}^{n}$, and any measurable set $B \subseteq D$, where $\eta \triangleq \eta_{1}+\theta\left(\eta_{1}, \eta_{2}, t\right)$, and where $\eta_{1}: \Omega \rightarrow \mathbb{R}^{n}$ and $\eta_{2}: \Omega \rightarrow \mathbb{R}^{m}$ are independent random vectors such that $\eta_{2}$ has the distribution described by the measure $\Pi(\cdot) / \Pi\left(\mathbb{R}^{m}\right)$, and $\mathbf{P}\left(\eta_{1} \in B\right)=\ell_{n}(B) \ell_{n}(D)^{-1}$. 
Notice that Condition 3.1 is satisfied if, for example, $\theta(x, u, t) \equiv \theta(u, t)$, that is, does not depend on $x$. Another example is described in the following proposition.

Proposition 3.2. Let there exist an integer $K>0, p_{1}, \ldots, p_{K} \in \mathbb{R}$ and $u_{1}, \ldots, u_{K} \in \mathbb{R}^{m}$ such that

$$
\int_{\mathbb{R}^{m}} \xi(u) \Pi(d u)=\sum_{i=1}^{K} \xi\left(u_{i}\right) p_{i}
$$

for any $\xi(\cdot) \in C\left(\mathbb{R}^{m}\right)$. Set $F_{i}(x, t) \triangleq x+\theta\left(x, u_{i}, t\right), D_{i} \triangleq F_{i}\left(\mathbb{R}^{n}, t\right)$. Let there exists the inverse function $F^{-1}(x, t): D_{i} \rightarrow \mathbb{R}^{n}$ for any given $t$ (i.e., $\left.F^{-1}(F(x, t), t) \equiv x\right)$. Moreover, let there exists a constant $c>0$ such that $\ell_{n}\left(F_{i}^{-1}(B, t)\right) \leq c \ell_{n}(B)$ for any measurable set $B \subset D_{i}, i=1,2, \ldots, n$. Then Condition 3.1 is satisfied.

Proof. Let $D \subset \mathbb{R}^{n}, \eta$ and $\eta_{1}$ be such as in Condition 3.1. For any measurable set $B \subset D$, we have

$$
\begin{aligned}
\mathbf{P}(\eta \in B) & =\sum_{i=1}^{n} p_{i} \mathbf{P}\left(F_{i}\left(\eta_{1}\right) \in B\right)=\sum_{i=1}^{n} p_{i} \mathbf{P}\left(\eta_{1} \in F_{i}^{-1}(B)\right) \\
& =\frac{\ell_{n}\left(F_{i}^{-1}(B)\right)}{\ell_{n}(D)} \leq c \frac{\ell_{n}(B)}{\ell_{n}(D)} .
\end{aligned}
$$

This completes the proof.

The following proposition will be useful.

Proposition 3.3 (see [3]). (i) If $\xi \in H^{1}$ and $\eta \in H^{0}$, then $\xi \eta \in \mathscr{W}$ and $\|\xi \eta\|_{\text {W }} \leq$ $c\|\xi\|_{H^{1}}\|\eta\|_{H^{0}}$, where $c=c(n, \mu)$ is a constant.

(ii) If $\xi \in H^{1}$ and $g \in \mathscr{W}^{*} \cap H^{0}$, then $\xi g \in H^{-1}$ and $\|\xi g\|_{H^{-1}} \leq c\|\xi\|_{H^{1}}\|g\|_{W^{*}}$ for $a$ constant $c=c(n, \mu)$.

Introduce the following parameter:

$$
\mathscr{P} \triangleq\left\{n, T, \delta, \sup _{x, t}|f(x, t)|, \sup _{x, t}|\beta(x, t)|, \sup _{x, t, i}\left|\frac{\partial \beta(x, t)}{\partial x_{i}}\right|, \Pi\left(\mathbb{R}^{m}\right), c_{\pi}\right\} .
$$

Theorem 3.4. Let Condition 3.1 be satisfied. Let $g \in \mathscr{X}, \varphi \in X^{-1}$, and $R \in H^{0}$ be given. Let $g_{\varepsilon} \in L_{\infty}(Q) \cap \mathscr{X}$ be such that $\left\|g_{\varepsilon}-g\right\|_{\mathscr{X}} \rightarrow 0$ as $\varepsilon \rightarrow 0+$. Then,

(i) for any $\varepsilon>0$, there exists the unique solution $V=V_{\varepsilon} \in Y_{1}$ of the problem (3.5) with $g=g_{\varepsilon}$;

(ii) the sequence $V_{\varepsilon}$ has a limit $V$ in $Y^{1}$ as $\varepsilon \rightarrow 0+$. This limit is uniquely defined by $\varphi, g$, and $V$ does not depend on the sequence $\left\{g_{\varepsilon}\right\}$. Moreover, $g V \in X^{-1}$ and

$$
\|V\|_{Y^{1}} \leq c\left(\|\varphi\|_{X^{-1}}+\|R\|_{H^{0}}\right),
$$

where $c>0$ is a constant which depends only on the parameters $\mathscr{P}, \mu$, and $\|g\|_{\mathscr{Q}}$.

REMARK 3.5. It can be seen from the proof of Theorem 3.4 that this theorem holds even if the derivatives $\partial f(x, t) / \partial x$ and $\partial \theta(x, t) / \partial x$ do not exist.

Definition 3.6. The limit $V$, defined in Theorem 3.4, is said to be the solution in $Y^{1}$ of the problem (3.5) with $g \in \mathscr{X}, \varphi \in X^{-1}$ and $R \in H^{0}$. 
Note that $V$ depends linearly on $(\varphi, R)$ for any given $g$. Moreover, by (3.10), it follows that $V=0$ if $\varphi=0$ and $R=0$. Hence it follows that the operator assigning the solution $V$ to the pair $(\varphi, R) \in X^{-1} \times H^{0}$ is also linear and homogeneous.

Definition 3.7. For every $g \in \mathscr{X}$, define the linear continuous operators $L(g)$ : $Y^{-1} \rightarrow Y^{1}$ and $\mathscr{L}(g): H^{0} \rightarrow Y^{1}$ such that $V=L(g) R$ for $V$ which is the solution in $Y^{1}$ of the problem (3.5) with $g \in \mathscr{X}, \varphi=0$, and $R \in H^{0}$, and $V=\mathscr{L}(g) R$ for $V$ which is the solution in $Y^{1}$ of the problem (3.5) with $g \in \mathscr{X}, \varphi \in X^{-1}$ and $R=0$.

The fact that these operators are continuous follows immediately from Theorem 3.4. Clearly, $V=L(g) \varphi+\mathscr{L}(g) R$ for $V$ which is the solution in $Y^{1}$ of the problem (3.5) with $g \in \mathscr{X}, \varphi \in X^{-1}$, and $R \in H^{0}$.

To prove Theorem 3.4, we need first a preliminary lemma.

LEMмA 3.8. Let $\varepsilon>0$ be such that there exists a solution $V=V_{\varepsilon} \in Y_{1}$ of the problem (3.5) with $g=g_{\varepsilon}$. Then

$$
\left\|V_{\varepsilon}\right\|_{Y^{1}} \leq c\left(\|\varphi\|_{X^{-1}}+\|R\|_{H^{0}}\right),
$$

where $c>0$ is a constant which depends only on the parameters $\mathscr{P}, \mu$, and $\|g\|_{\mathscr{Q}}$.

Proof OF Lemma 3.8. We use below the elementary estimate $u v \leq u^{2} /(2 \gamma)+$ $v^{2} \gamma 2$ (for all $u, v, \gamma \in \mathbb{R}, \gamma>0$ ).

Let $v \in H^{1} \cap C^{2}\left(\mathbb{R}^{n}\right)$. For $t \in[0, T]$, we have

$$
\begin{aligned}
\left(v, \mathscr{A}_{C}(t) v\right)_{H^{0}}= & \left(v, \sum_{i, j=1}^{n} b_{i j} \frac{\partial^{2} v}{\partial x_{i} \partial x_{j}}\right)_{H^{0}}+\left(v, \sum_{i=1}^{n} \hat{f}_{i} \frac{\partial v}{\partial x_{i}}\right)_{H^{0}} \\
= & -\sum_{i, j=1}^{n}\left(\frac{\partial v}{\partial x_{i}}, b_{i j} \frac{\partial v}{\partial x_{j}}\right)_{H^{0}}-\sum_{i, j=1}^{n}\left(v, \frac{\partial b_{i j}}{\partial x_{i}} \frac{\partial v}{\partial x_{j}}\right)_{H^{0}}+\sum_{i=1}^{n}\left(v, \hat{f}_{i} \frac{\partial v}{\partial x_{i}}\right)_{H^{0}} \\
\leq & -\delta \sum_{i=1}^{n}\left\|\frac{\partial v}{\partial x_{i}}\right\|_{H^{0}}^{2}+\sum_{i, j=1}^{n}\|v\|_{H^{0}}\left\|\frac{\partial b_{i j}}{\partial x_{i}}\right\|_{L_{\infty}(Q)}\left\|\frac{\partial v}{\partial x_{j}}\right\|_{H^{0}} \\
& +\sum_{i=1}^{n}\|v\|_{H^{0}}\left\|\hat{f}_{i}\right\|_{L_{\infty}(Q)}\left\|\frac{\partial v}{\partial x_{i}}\right\|_{H^{0}} \\
\leq & -\delta \sum_{i=1}^{n}\left\|\frac{\partial v}{\partial x_{i}}\right\|_{H^{0}}^{2}+\frac{\delta}{4} \sum_{i=1}^{n}\left\|\frac{\partial v}{\partial x_{i}}\right\|_{H^{0}}^{2} \\
& +\frac{C}{\delta}\|v\|_{H^{0}}^{2} \sum_{i, j=1}^{n}\left(\left\|\frac{\partial b_{i j}}{\partial x_{i}}\right\|_{L_{\infty}(Q)}^{2}+\left\|f_{i}\right\|_{L_{\infty}(Q)}^{2}\right)
\end{aligned}
$$

where $C=C(n)$ is a constant. Hence we obtain the inequality

$$
\left(v, \mathscr{A}_{C}(t) v\right)_{H^{0}} \leq-\frac{3 \delta}{4} \sum_{i=1}^{n}\left\|\frac{\partial v}{\partial x_{i}}\right\|_{H^{0}}^{2}+C_{1}^{\prime}\|v\|_{H^{0}}^{2}
$$

for all $v \in H^{1}, t \in[0, T]$, where a constant $C_{1}^{\prime}$ depends only on $\mathscr{P}$. 
Let $D_{K} \triangleq\left\{x \in \mathbb{R}^{n}:|x| \leq K\right\}$. By Condition 3.1,

$$
\begin{aligned}
\left\|g^{\prime}(t) v\right\|_{H^{0}}^{2} & =\int_{\mathbb{R}^{n}} d x\left|\int_{\mathbb{R}^{m}} v(x+\theta(t, x, u)) \Pi(d u)\right|^{2} \\
& \leq \int_{\mathbb{R}^{n}} d x \int_{\mathbb{R}^{m}}|v(x+\theta(t, x, u))|^{2} \Pi(d u) \\
& =\lim _{K \rightarrow+\infty} \int_{D_{K}} d x \int_{\mathbb{R}^{m}}|v(x+\theta(t, x, u))|^{2} \Pi(d u) \\
& =\lim _{K \rightarrow+\infty} \ell_{n}\left(D_{K}\right) \int_{\mathbb{R}^{n}} \mu_{K}(d y)|v(y)|^{2} \\
& \leq c_{\pi} \int_{\mathbb{R}^{n}}|v(y)|^{2} d y=c_{\pi}\|v\|_{H^{0}}^{2},
\end{aligned}
$$

where $\mu_{K}(\cdot)$ is the probability measure which describes the distribution of a random vector $\eta=\eta_{K}$ such as in Condition 3.1, where $D=D_{K}$. Then

$$
\|\mathscr{f}(t) v\|_{H^{0}}^{2} \leq\left(c_{\pi}+\Pi\left(\mathbb{R}^{m}\right)\right)\|v\|_{H^{0}}^{2} .
$$

Thus,

$$
(v, \mathcal{A}(t) v)_{H^{0}} \leq-\frac{3 \delta}{4} \sum_{i=1}^{n}\left\|\frac{\partial v}{\partial x_{i}}\right\|_{H^{0}}^{2}+C_{1}\|v\|_{H^{0}}^{2}
$$

for all $v \in H^{1}$ and $t \in[0, T]$, where a constant $C_{1}^{\prime}$ depends only on $\mathscr{P}$.

Furthermore, we have

$$
\begin{aligned}
\left(v, \varphi_{\varepsilon}(\cdot, t)\right)_{H^{0}} & \leq\|v\|_{H^{1}}\left\|\varphi_{\varepsilon}(\cdot, t)\right\|_{H^{-1}} \\
& \leq \frac{\delta}{4}\left(\sum_{i=1}^{n}\left\|\frac{\partial v}{\partial x_{i}}\right\|_{H^{0}}^{2}+\|v\|_{H^{0}}^{2}\right)+C_{2}\left\|\varphi_{\varepsilon}(\cdot, t)\right\|_{H^{-1}}^{2}
\end{aligned}
$$

for all $v \in H^{1}$ and $t \in[0, T]$, where a constant $C_{2}$ also depends only on $\mathscr{P}$.

Proposition 3.3(i) yields

$$
\begin{aligned}
\left(v, g_{\varepsilon} v\right)_{H^{0}} & \leq\left\|v^{2}\right\|\left\|_{W}\right\| g_{\varepsilon}\left\|_{W^{*}} \leq C_{3}\right\| v\left\|_{H^{1}}\right\| v\left\|_{H^{0}}\right\| g_{\varepsilon} \|_{W^{*}} \\
& \leq \frac{\delta}{4} \sum_{i=1}^{n}\|v\|_{H^{1}}^{2}+\hat{C}_{3}\|v\|_{H_{0}}^{2} \\
& \leq \frac{\delta}{4} \sum_{i=1}^{n}\left\|\frac{\partial v}{\partial x_{i}}\right\|_{H^{0}}^{2}+C_{3}\|v\|_{H_{0}}^{2} \quad \forall v \in H^{1}
\end{aligned}
$$

where constants $\hat{C}_{3}$ and $C_{3}$ depend on $\left\|g_{\varepsilon}\right\|_{\mathcal{W}^{*}}, \delta$, and $n$.

For the solution $V=V_{\varepsilon}$ of the problem (3.5) with $g=g_{\varepsilon}, \varepsilon \in\left(0, \varepsilon_{1}\right]$, we have from (3.12), (3.15), (3.16), (3.17), and (3.18) that

$$
\begin{aligned}
\left\|V_{\varepsilon}(\cdot, t)\right\|_{H^{0}}^{2} & -\left\|V_{\varepsilon}(\cdot, T)\right\|_{H^{0}}^{2} \\
& =2 \int_{t}^{T}\left(V_{\varepsilon}(\cdot, s), A V_{\varepsilon}(\cdot, s)+g_{\varepsilon} V_{\varepsilon}(\cdot, s)+\varphi(\cdot, s)\right)_{H^{0}} d s \\
& \leq \int_{t}^{T}\left\{-\delta \sum_{i=1}^{n}\left\|\frac{\partial V_{\varepsilon}}{\partial x_{i}}(\cdot, s)\right\|_{H^{0}}^{2}+C_{4}\left(\left\|V_{\varepsilon}(\cdot, s)\right\|_{H^{0}}^{2}+\|\varphi(\cdot, s)\|_{H^{-1}}^{2}\right)\right\} d s,
\end{aligned}
$$


where a constant $C_{4}$ depends on $\mathscr{P}, \mu$, and $\|g\|_{\mathscr{X}}$. Thus,

$$
\left\|V_{\varepsilon}\right\|_{Y^{1}} \leq C_{*}\left(\|\varphi\|_{X^{-1}}+\|R\|_{H^{0}}\right) \quad \forall \varepsilon \in\left(0, \varepsilon_{1}\right]
$$

where a constant $C_{*}$ also depends on $\mathscr{P}, \mu$, and $\|g\|_{\mathscr{X}}$. This completes the proof of Lemma 3.8.

Proof of Theorem 3.4. We prove (i). Consider a boundary value problem

$$
\frac{\partial V}{\partial t}+\mathscr{A}_{C} V+g_{\varepsilon} V=-\varphi, \quad V(x, T)=R(x) .
$$

The solution $V \in Y^{1}$ of this problem is well defined. For every $g \in L_{\infty}(Q)$, introduce linear continuous operators $L_{c}(g): X^{-1} \rightarrow Y^{1}$ and $\mathscr{L}_{c}(g): H^{0} \rightarrow Y^{1}$ such that $V=$ $L_{c}(g) \varphi+\mathscr{L}_{c}(g) R$ for $V$ which is the solution in $Y^{1}$ of the problem (3.21) with given $g$, $\varphi$, and $R$.

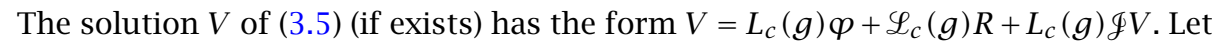

$$
\begin{gathered}
V_{0} \triangleq 0 \in Y^{1}, \\
V_{k} \triangleq L_{c}(g) \varphi+\mathscr{L}_{c}(g) R+L_{c}(g) \mathscr{g} V_{k-1}, \quad U_{k} \triangleq V_{k}-V_{k-1}, \quad k=1,2, \ldots .
\end{gathered}
$$

It suffices to prove that $U_{k} \rightarrow 0$ in $Y^{1}$ as $k \rightarrow+\infty$. Set

$$
y_{k}(t) \triangleq\left\|U_{k}(\cdot, t)\right\|_{H^{0}}+\delta \sum_{i=1}^{n} \int_{t}^{T}\left\|\frac{\partial U_{k}}{\partial x_{i}}(\cdot, s)\right\|_{H^{0}}^{2} d s .
$$

Similar to (3.19), we have

$$
y_{k}(t) \leq c_{1}+C_{4} \int_{t}^{T}\left(y_{k}(s)+y_{k-1}(s)\right) d s
$$

where $c_{1} \triangleq\|R(\cdot)\|_{H^{0}}$. By the Bellman inequality,

$$
y_{k}(t) \leq c_{1} e^{C_{4}(T-t)} \int_{t}^{T} y_{k-1}(s) d s .
$$

It is easy to see that $y_{k}(t) \leq C^{k}$, where $C>0$ is a constant independent of $k$ and $t$. After standard iterations, we have that $\sup _{t \in[0, T]} y_{k}(t) \rightarrow 0$ as $k \rightarrow+\infty$. Thus, $\left\{V_{k}\right\}$ is a Cauchy sequence in $Y^{1}$. Then (i) follows.

We show that the sequence $\left\{V_{\varepsilon}\right\}, \varepsilon \rightarrow 0$, is a Cauchy sequence in the space $Y^{1}$. Let $\varepsilon_{1} \rightarrow 0, \varepsilon_{2} \rightarrow 0$ and let $W=V_{\varepsilon_{1}}-V_{\varepsilon_{2}}$, then

$$
\frac{\partial W}{\partial t}+\mathscr{A} W+g_{\varepsilon_{1}} W=-\xi, \quad W(x, T)=0,
$$

where $\xi \triangleq\left(g_{\varepsilon_{1}}-g_{\varepsilon_{2}}\right) V_{\varepsilon_{2}}$. Furthermore,

$$
\left\|g_{\varepsilon_{1}}-g_{\varepsilon_{2}}\right\|_{W^{*}} \longrightarrow 0
$$


because $\left\{g_{\varepsilon}\right\}$ is a Cauchy sequence. By Proposition 3.3(ii) and (3.20), (3.27),

$$
\begin{aligned}
\left\|\left(g_{\varepsilon_{1}}-g_{\varepsilon_{2}}\right) V_{\varepsilon_{2}}\right\|_{X^{-1}} & =\int_{0}^{T}\left\|\left(g_{\varepsilon_{1}}-g_{\varepsilon_{2}}\right) V_{\varepsilon_{2}}(\cdot, t)\right\|_{H^{-1}} d t \\
& \leq \int_{0}^{T}\left\|g_{\varepsilon_{1}}-g_{\varepsilon_{2}}\right\|_{W^{*}}\left\|V_{\varepsilon_{2}}(\cdot, t)\right\|_{H^{1}} d t \\
& \leq\left\|g_{\varepsilon_{1}}-g_{\varepsilon_{2}}\right\|_{\mathscr{X}} \int_{0}^{T}\left\|V_{\varepsilon_{2}}(\cdot, t)\right\|_{H^{1}} d t \\
& =\left\|g_{\varepsilon_{1}}-g_{\varepsilon_{2}}\right\|_{W^{*}}\left\|V_{\varepsilon_{2}}\right\|_{X^{1}} \longrightarrow 0
\end{aligned}
$$

as $\varepsilon_{1} \rightarrow 0, \varepsilon_{2} \rightarrow 0$. Hence $\|\xi\|_{X^{-1}} \rightarrow 0$. The estimate (3.20) applied to the solution $W$ of the boundary value problem (3.26) yields

$$
\|W\|_{Y^{1}} \leq C_{*}\|\xi\|_{X^{-1}} \longrightarrow 0 .
$$

Hence the sequence $\left\{V_{\varepsilon}\right\}, \varepsilon \rightarrow 0$, is a Cauchy sequence (and has a limit) in the Banach space $Y^{1}$. The estimate (3.10) and the uniqueness of $V$ follows from (3.20) and (3.26). This completes the proof of Theorem 3.4.

COROLLARY 3.9. Let $V_{\varepsilon} \triangleq L\left(g_{\varepsilon}\right) \varphi_{\varepsilon}+\mathscr{L}\left(g_{\varepsilon}\right) R_{\varepsilon}$, and $V \triangleq L(g) \varphi+\mathscr{L}(g) R$, where $g, g_{\varepsilon} \in$ $\mathscr{X}, \varphi, \varphi_{\varepsilon} \in X^{-1}$ are such that $\left\|g_{\varepsilon}-g\right\|_{\mathscr{X}} \rightarrow 0,\left\|\varphi_{\varepsilon}-\varphi\right\|_{X^{-1}} \rightarrow 0$ and $\left\|R_{\varepsilon}-R\right\|_{H^{0}} \rightarrow 0$ as $\varepsilon \rightarrow 0+$. Then $\left\|V_{\varepsilon}-V\right\|_{Y^{1}} \rightarrow 0$.

Proof. For the sake of simplicity, assume that $\varphi_{\varepsilon} \equiv \varphi$. Let $\left\|\mathscr{L}\left(g_{\varepsilon}\right)\right\|$ denote the norm of the operator $\mathscr{L}\left(g_{\varepsilon}\right): H^{0} \rightarrow Y^{1}$. By Theorem $3.4, \sup _{\varepsilon}\left\|\mathscr{L}\left(g_{\varepsilon}\right)\right\| \leq$ const. Then

$$
\begin{aligned}
\left\|V_{\varepsilon}-V\right\|_{Y^{1}} & \leq\left\|\mathscr{L}\left(g_{\varepsilon}\right) R_{\varepsilon}-\mathscr{L}\left(g_{\varepsilon}\right) R\right\|_{Y^{1}}+\left\|\mathscr{L}\left(g_{\varepsilon}\right) R-\mathscr{L}(g) R\right\|_{Y^{1}} \\
& \leq \sup _{\varepsilon}\left\|\mathscr{L}\left(g_{\varepsilon}\right)\right\|\left\|R_{\varepsilon}-R\right\|_{H^{0}}+\left\|\mathscr{L}\left(g_{\varepsilon}\right) R-\mathscr{L}(g) R\right\|_{Y^{1}} .
\end{aligned}
$$

By Theorem 3.4, it also follows that $\left\|\mathscr{L}\left(g_{\varepsilon}\right) R-\mathscr{L}(g) R\right\|_{Y^{1}} \rightarrow 0$ as $\varepsilon \rightarrow 0$. Then the proof follows.

4. Adjoint equations. Let $\mathscr{A}_{c}^{*}=\mathscr{A}_{c}^{*}(t)$ be the operator which is formally adjoint to the operator $\mathscr{A}_{\mathcal{C}}(t)$ defined by (3.2),

$$
A_{c}^{*}(t) p=\sum_{i, j=1}^{n} \frac{\partial^{2}}{\partial x_{i} \partial x_{j}}\left(b_{i j}(x, t) p(x)\right)-\sum_{i=1}^{n} \frac{\partial}{\partial x_{i}}\left(\hat{f}_{i}(x, t) p(x)\right) .
$$

Let $\mathscr{F}^{*}=\mathscr{F}^{*}(t): H^{0} \rightarrow H^{0}$ be the operator which is adjoint to the operator $\mathscr{E}=\mathscr{F}(t)$ : $H^{0} \rightarrow H^{0}$ defined by (3.3). Let $\mathscr{A}^{*} \triangleq \mathscr{A}_{c}^{*}+\mathscr{J}^{*}$. Consider the following boundary value problem in $Q$ :

$$
\frac{\partial p}{\partial t}=\mathscr{A}^{*} p+g p+\varphi, \quad p(x, 0)=\rho(x) .
$$

THEOREM 4.1. Assume that Condition 3.1 is satisfied. Let $g \in \mathscr{X}, \varphi \in X^{-1}$, and $\rho \in H^{0}$ be given. Let $g_{\varepsilon} \in L_{\infty}(Q) \cap \mathscr{X}$ be such that

$$
\left\|g_{\varepsilon}-g\right\|_{\mathscr{X}} \longrightarrow 0 \text { as } \varepsilon \longrightarrow 0+.
$$


Then,

(i) for any $\varepsilon>0$, there exist the unique solution $p_{\varepsilon}$ of (4.2) with $g=g_{\varepsilon}$;

(ii) the sequence $p_{\varepsilon}$ has a unique limit $p$ in $Y^{1}$ as $\varepsilon \rightarrow 0+$, and

$$
\|p\|_{Y^{1}} \leq c\left(\|\varphi\|_{X^{-1}}+\|\rho\|_{H^{0}}\right)
$$

where a constant $c>0$ depends only on the parameters $\mathscr{P}, \mu$, and $\|g\|_{\mathscr{x}}$.

The proof of Theorem 4.1 is similar to the proof of Theorem 4.4. Note only that, by Remark 3.5, it follows that the coefficients of the operator $\mathscr{A}_{C}^{*}$ are smooth enough, and, by (3.15), $\left\|\mathscr{F}^{*} v\right\|_{H^{0}}^{2} \leq\left(c_{\pi}+\Pi\left(\mathbb{R}^{m}\right)\right)\|v\|_{H^{0}}^{2}$ for all $v \in H^{0}$.

For $g \in \mathscr{X}$, introduce a linear continuous operators $\hat{\mathbf{L}}(g): X^{-1} \rightarrow H^{0}$ and $\mathbf{L}_{0}(g)$ : $H^{0} \rightarrow H^{0}$ such that $V(\cdot, 0)=\hat{\mathbf{L}}(g) \varphi+\mathbf{L}_{T}(g) R$, where $V=L(g) \varphi+\mathscr{L} R$ is the solution of the problem (3.5).

Proposition 4.2. For $p, g$, $\varphi$, and $\rho$ from Theorem $4.1, p=L(g)^{*} \varphi+\hat{\mathbf{L}}(g)^{*} \rho$ and $p(\cdot, T)=L(g)^{*} \varphi+\mathbf{L}_{T}(g)^{*} \rho$, where $L(g)^{*}: X^{-1} \rightarrow X^{1}, \hat{\mathbf{L}}(g)^{*}: H^{0} \rightarrow X^{1}$ and $\mathbf{L}_{T}(g)^{*}:$ $H^{0} \rightarrow H^{0}$ are linear continuous operators which are adjoint to the operators $L(g)$ : $X^{-1} \rightarrow X^{1}$ and $\hat{\mathbf{L}}(g): X^{-1} \rightarrow H^{0}$ and $\mathbf{L}_{T}(g): H^{0} \rightarrow H^{0}$ correspondingly.

Proof. Let $\phi \in X^{0}, R \in H^{0}$ be arbitrary, $V=L(g) \phi+\mathscr{L}(g) R$. Then

$$
\begin{aligned}
(p(\cdot, T), R)_{H^{0}}-\left(\rho, \hat{\mathbf{L}}(g) \phi+\mathbf{L}_{T} R\right)_{H^{0}} \\
=(p(\cdot, T), V(\cdot, T))_{H^{0}}-(p(\cdot, 0), V(\cdot, 0))_{H^{0}} \\
=\left(\frac{\partial p}{\partial t}, V\right)_{X^{0}}+\left(p, \frac{\partial V}{\partial t}\right)_{X^{0}} \\
=\left(\mathscr{A}^{*} p+g p+\varphi, V\right)_{X^{0}}+(p,-\mathscr{A} V-g V-\phi)_{X^{0}} \\
=(\varphi, V)_{X^{0}}-(p, \phi)_{X^{0}} \\
=(\varphi, L(g) \phi+\mathscr{L}(g) R)_{X^{0}}-(p, \phi)_{X^{0}} .
\end{aligned}
$$

Then

$$
(p(\cdot, T), R)_{H^{0}}+(p, \phi)_{X^{0}}=\left(\rho, \hat{\mathbf{L}}(g) \phi+\mathbf{L}_{T} R\right)_{H^{0}}+(\varphi, L(g) \phi+\mathscr{L}(g) R)_{X^{0}} .
$$

Then the proof follows.

Condition 4.3. There exists uniformly bounded derivatives $\partial^{k} \beta(x, u, t) / \partial x^{k}$, $\partial^{k} f(x, t) / \partial x^{k}$, and $\partial^{k} \theta(x, u, t) / \partial x^{k}$ for $k=1,2$.

THEOREM 4.4. Let Conditions 3.1 and 4.3 be satisfied, let $g(x, t): Q \rightarrow \mathbb{R}$ be a Borel measurable function which belongs to $\mathscr{X}$ and is bounded together with the derivatives $\partial^{k} g(x, t) / d x^{k}$ for $k=1,2$. Let the vector a in (2.1) have the probability density function $\rho \in H^{0}$, and let $p \triangleq \hat{\mathbf{L}}(g)^{*} \rho$. Then

$$
\mathbf{E} R(y(T)) \exp \left(\int_{0}^{T} g(y(t), t) d t\right)=\int_{\mathbb{R}^{n}} p(x, T) R(x) d x
$$

for all Borel measurable $R(\cdot) \in H^{0}$. In particular, if $g=0$ then $p(x, t)$ is the probability density function of the solution $y(t)$ of (2.1). 
Proof. It suffices to prove (4.7) with $R(\cdot) \in H^{0} \cap C^{2}\left(\mathbb{R}^{n}\right)$. For $(x, s) \in Q$, set $V(x, s)$ $\triangleq \mathbf{E}\{R(y(T)) \mid y(s)=x\}$. By [6, Theorem 4, page 296], it follows that $V=\mathbf{L}(0) R$. By Proposition 4.2, it follows that

$$
\mathbf{E} R(y(T))=(V(\cdot, 0), \rho)_{H^{0}}=\left(\mathbf{L}_{T} R, \rho\right)_{H^{0}}=\left(R, \mathbf{L}_{T}^{*} \rho\right)_{H^{0}}=(R, p(\cdot, T))_{H^{0}}
$$

for all $R(\cdot) \in H^{0} \cap C^{2}\left(\mathbb{R}^{n}\right)$. This completes the proof.

COROLlary 4.5. Let $V=\mathscr{L}(g) R$, where $R \in H^{0}$. Then there exist a version of $V$ such that $\operatorname{essup}_{x, t} V(x, t) \leq \max _{x} R(x)$ and $\operatorname{essinf}_{x, t} V(x, t) \geq \min _{x} R(x)$.

Proof. If $R(\cdot) \in H^{0} \cap C^{2}\left(\mathbb{R}^{n}\right)$ then $V(x, s)=\mathbf{E}\{R(y(T)) \mid y(s)=x\}$ and the proof follows. For the general case $R \in H^{0}$, the proof can be obtained by a standard approximation.

Condition 4.6. (i) There exist uniformly bounded derivatives $\partial^{m} \beta(x, u, t) / \partial x^{m}$ for $m \leq 4, \partial^{l} f(x, t) / \partial x^{l}$ for $l=1,2,3$, and $\partial^{k} \theta(x, u, t) / \partial x^{k}$ for $k=1,2$.

(ii) There exist $c_{*} \in \mathbb{R}$, a measure $\Pi_{*}(\cdot)$ in $\mathbb{R}^{m}$, and a bounded and Borel measurable function $\theta_{*}(x, u, t): \mathbb{R}^{n} \times \mathbb{R}^{m} \times \mathbb{R} \rightarrow \mathbb{R}^{n \times n}$ which is continuous in $u$, such that $\Pi_{*}\left(\mathbb{R}^{m}\right)<+\infty$ and $\mathscr{F}^{*}(t) v=\int_{\mathbb{R}^{m}} v\left(x+\theta_{*}(x, u, t)\right) \Pi_{*}(d u)+c_{*} v$ for any $v \in H^{0}$.

(iii) The derivatives $\partial^{k} \theta_{*}(x, u, t) / \partial x^{k}$ are bounded for $k=1,2$ and Condition 3.1 is satisfied with substituting $(\Pi(\cdot), \theta(\cdot))=\left(\Pi_{*}(\cdot), \theta_{*}(\cdot)\right)$.

Note that if the mapping $z=x+\theta(x, u, t)$ maps $\mathbb{R}^{n}$ one-to-one onto itself for any $(u, t)$, then $\theta_{*}(\cdot)$ can be found such that $x=z-\theta_{*}(z, u, t)$ is the inverse mapping. If the last one is differentiable, then $\Pi_{*}(\cdot)$ can be found as $\Pi_{*}(d x)=J(x) \Pi(d x)$, where $J(x)$ is the Jacobian of the transformation $y=x-\theta_{*}(x, u, t)$ (see [6, page 299]).

Corollary 4.7. Let Conditions 3.1, 4.3, and 4.6 be satisfied. Let $\rho \in L_{\infty}\left(\mathbb{R}^{n}\right) \cap H^{0}$, and let $p \triangleq \hat{\mathbf{L}}(0)^{*} \rho$. Then $p \in L_{\infty}(Q)$.

Proof. It can be seen that equation (4.7) after a change of time variable can be rewritten in the form (3.2), and then Corollary 4.5 is satisfied. This completes the proof.

5. On a class of acceptable hypersurfaces. We will use the equations from Sections 3 and 4 for the distributions of the occupation time on hypersurfaces. In this section we describe a class of acceptable hypersurfaces.

Let $\Gamma \subset D$ be some $(n-1)$-dimensional hypersurface. By $e^{(i)}$ we denote the $i$ th unit vectors in $\mathbb{R}^{n}, i=1, \ldots, n$. Let $\mathbf{n}(x)$ be the normal to $\Gamma$ in $x$, and let $\alpha_{i}(x)$ be the angle between $e^{(i)}$ and $\mathbf{n}(x)$.

Introduce the functions $\gamma_{i}: \Gamma \rightarrow \mathbb{R}, i=1, \ldots, n$, such that

$$
\gamma_{i}(x)= \begin{cases}\left|\cos \alpha_{i}(x)\right| & \text { if the normal } \mathbf{n}(x) \text { at } x \text { is uniquely defined, } \\ 0 & \text { if the normal at } x \text { is not defined. }\end{cases}
$$

(In fact, $\mathbf{n}(x)$ is not defined at points of violation of smoothness of $Г$.) 
Denote by $N(x, j, \Gamma)$ the number of intersections of the hypersurface $\Gamma$ with the ray from $x=\left(x_{1}, x_{2}, \ldots, x_{n}\right)$ to $\left(x_{1}, \ldots, x_{j-1},-\infty, x_{j+1}, \ldots, x_{n}\right)$. Let $\hat{x}_{k}(x, j)$ be the corresponding intersection points.

We assume that $N(x, j, \Gamma)=+\infty$, if the ray is tangential to $\Gamma$.

Set

$$
\begin{aligned}
G_{j}(x) & \triangleq \sum_{k=1}^{N(x, j, \Gamma)} \gamma_{j}\left(\hat{x}_{k}(x, j)\right), \quad g=\sum_{j=1}^{n} \frac{\partial G_{j}}{\partial x_{j}}, \\
\Gamma(\varepsilon) & \triangleq\left\{x \in \mathbb{R}^{n}: \inf _{y \in \Gamma}|x-y| \leq \frac{\varepsilon}{2}\right\}, \\
g_{\varepsilon}(x) & \triangleq \frac{1}{\varepsilon} \operatorname{Ind}\{x \in \Gamma(\varepsilon)\} .
\end{aligned}
$$

Definition 5.1. A set $\hat{\Gamma} \in \mathbb{R}^{n}$ is said to be an $(n-1)$-dimensional polyhedron if there exist an integer $N$ and $c_{i} \in \mathbb{R}^{n}, \delta_{i} \in \mathbb{R}, i=0,1, \ldots, N$ such that $\hat{\Gamma}=\left\{x \in \mathbb{R}^{n}\right.$ : $\left.c_{0}^{\prime} x=\delta_{0}, c_{i}^{\prime} x \leq \delta_{i}, i=1, \ldots, N\right\}$. The set $\left\{x \in \mathbb{R}^{n}: c_{0}^{\prime} x=\delta_{0}, c_{i}^{\prime} x<\delta_{i}, i=1, \ldots, N\right\}$ is said to be the interior of $\hat{\Gamma}$.

LEMMA 5.2 (see [3]). Let a hypersurface $\Gamma \subset \mathbb{R}^{n}$ be bounded and such that there exists a set $\hat{\Gamma} \subset \mathbb{R}^{n}$ and a continuous bijection $\mu: \mathbb{R}^{n} \rightarrow \mathbb{R}^{n}$ which satisfy the following assumptions:

(i) $\Gamma=\mu(\hat{\Gamma})$;

(ii) $\hat{\Gamma}=\cup_{i=1}^{\mathcal{N}} \hat{\Gamma}_{i}$, where $\mathcal{N}$ is an integer, $\hat{\Gamma}_{i}$ is $(n-1)$-dimensional polyhedron;

(iii) $\mathcal{M}: \hat{\Gamma}_{i} \rightarrow \mathbb{R}^{n}$ are $C^{1}$-smooth bijections, $i=1, \ldots, \mathcal{N}$;

(iv) $\left|\mathbf{n}(x)-\mathbf{n}_{i}\right| \leq \delta_{0}$, if $\mathcal{M}^{-1}(x)$ belongs to the interior of $\hat{\Gamma}_{i}, i=1, \ldots, \mathcal{N}$, where $x \in \Gamma$, $\delta_{0} \leq n^{-2} / 2$ is a constant, $\mathbf{n}(x)$ is the normal to $\Gamma$ in $x$, and $\mathbf{n}_{i}$ is the normal to $\hat{\Gamma}_{i}$; it is assumed that the orientations of these normals are fixed and $|\mathbf{n}(x)|=1$, $\left|\mathbf{n}_{i}\right|=1$;

(v) $M(x)=x$, if $x$ is a top point of some $\hat{\Gamma}_{i}$.

Then $N(j, x, \Gamma)<+\infty$ for a.e. $x$. Moreover, $g \in W^{*} \cap H^{-1}$ and $g_{\varepsilon}(\cdot) \rightarrow g$ in $W^{*}$.

6. Existence of the occupation time density and an analog of Meyer-Tanaka formula. Set

$$
g_{\varepsilon}(x, t) \triangleq \frac{1}{\varepsilon} \operatorname{Ind}\{x \in \Gamma(\varepsilon, t)\}, \quad l_{\varepsilon}(t) \triangleq \int_{0}^{t} g_{\varepsilon}(y(s), s) .
$$

It is natural to interpret the limit of $l_{\varepsilon}(T)$ as the occupation time of $y(t)$ on $\Gamma(t)$.

Condition 6.1. (i) The hypersurface $\Gamma(t)$ is such that the assumptions of Lemma 5.2 hold for $\Gamma=\Gamma(t)$ for a.e. $t \in[0, T]$ and $g=g(t) \in X^{-1}$, where $g(t) \triangleq \lim _{\varepsilon \rightarrow 0} g_{\varepsilon}(t)$ (by Lemma 5.2, the limit exists in $H^{-1}$ for a.e. $t \in[0, T]$ ).

(ii) The initial vector $a=y(0)$ has probability density function $\rho \in L_{\infty}\left(\mathbb{R}^{n}\right)$.

(iii) The function $\beta(x, t)$ in (2.1) is continuous.

Note that the assumptions of Lemma 5.2 hold for disks, spheres, and many other piecewise $C^{1}$-smooth $(n-1)$-dimensional surfaces. Moreover, it can be easy to find examples when the surface $\Gamma(t)$ changes in time, approaching a fractal, but $g \in X^{-1}$. 
EXAMPLE 6.2. Let $n=2, T=2, \Gamma(t)=\left\{\left(x_{1}, x_{2}\right): x_{2}=\sin \left(x_{1}(1-t)^{-1 / 3}\right), x_{1} \in\right.$ $[-1,1]\}$. Then $N\left(\left(2, x_{2}\right), 2, \Gamma(t)\right) \equiv 1$ and

$$
\begin{aligned}
\|g\|_{X^{-1}}^{2} & =\int_{0}^{T}\|g(t)\|_{H^{-1}}^{2} d t \\
& \leq \mathrm{const} \int_{0}^{T}\left[1+\sup _{x_{2}} N\left(\left(2, x_{2}\right), 1, \Gamma(t)\right)^{2}\right] d t \\
& \leq \mathrm{const}\left(2+\int_{0}^{2}(1-t)^{-2 / 3} d t\right)<+\infty
\end{aligned}
$$

Hence $g=g(t) \in X^{-1}$.

The following example presents a fractal $\Gamma$ which is constant in time.

EXAMPLE 6.3. Let $n=2, \Gamma(t) \equiv \Gamma=\left\{\left(x_{1}, x_{2}\right): x_{2}=x_{1} \sin \left(x_{1}^{-1 / 3}\right), x_{1} \in[-1,1]\right\}$. Then $N\left(\left(2, x_{2}\right), 2, \Gamma(t)\right) \equiv 1$ and

$$
\|g\|_{H^{-1}}^{2} \leq\left(1+\int_{-1}^{1} d x_{2} N\left(\left(2, x_{2}\right), 1, \Gamma(t)\right)^{2}\right) \leq \mathrm{const}\left(1+\int_{-1}^{1} x_{1}^{-2 / 3} d x_{1}\right)<+\infty .
$$

Hence $g \in H^{-1}$.

Denote by $\beta_{j}$ the columns of the matrix $\beta, j=1, \ldots, n$. Let $\mathscr{F}_{t}$ be the filtration of complete $\sigma$-algebras of events, generated by $\left\{a, w(s), v(B, s), s \leq t, B \in \overline{\mathscr{P}}_{n}\right\}$.

Introduce the set $\tilde{\mathscr{y}}$ of all bounded functions $\xi(t)=\xi(t, \omega):[0, T] \times \Omega \rightarrow \mathbb{R}^{n}$ which are progressively measurable with respect to $\mathscr{F}_{t}$, and introduce the set $\tilde{\mathscr{E}}$ of all bounded functions $\psi(u, t)=\psi(u, t, \omega): \mathbb{R}^{n} \times[0, T] \times \Omega \rightarrow \mathbb{R}^{n}$ which are progressively measurable with respect to $\mathscr{F}_{t}$ for all $u$.

Introduce the Hilbert space $\mathscr{\mathscr { y }}_{2}$ as the completion of $\widetilde{y}$ with respect to the norm $\|\xi\|_{9_{2}} \triangleq \mathbf{E} \int_{0}^{T}|\xi(t)|^{2} d t$, and introduce the Hilbert space $\mathscr{L}_{2}$ as the completion of $\tilde{\mathscr{E}}$ with respect to the norm $\|\psi\|_{\mathscr{L}_{2}} \triangleq \mathbf{E} \int_{0}^{T} d t \int_{\mathbb{R}^{n}}|\psi(u, t)|^{2} \Pi(d u)$.

We present now an analog of the Meyer-Tanaka formula (cf. [9] or [11, page 169]).

THEOREM 6.4. Assume that Conditions 3.1, 4.3, 4.6, and 6.1 are satisfied. Let $V \triangleq$ $L(0) g$ (by definition, this $V$ belongs $Y^{1}$ ). Let $V$ and $\partial V / \partial x$ be Borel measurable representatives $V$ and $\partial V / \partial x$ of corresponding equivalence classes in $L_{2}(Q)$. Then

$$
\frac{\partial V}{\partial x}(y(t), t) \beta_{j}(y(t), t) \in \mathscr{Y}_{2}, \quad V(y(t)+\theta(y(t), u, t), t)-V(y(t), t) \in \mathscr{L}_{2},
$$

and $\mathbf{E}\left|l_{\varepsilon}(T)-\hat{\mathbf{t}}(T)\right|^{2} \rightarrow 0$ as $\varepsilon \rightarrow 0$, where

$$
\begin{aligned}
\hat{\mathbf{t}}(T) \triangleq & V(a, 0)+\sum_{j=1}^{n} \int_{0}^{T} \frac{\partial V}{\partial x}(y(t), t) \beta_{j}(y(t), t) d w_{j}(t) \\
& +\int_{0}^{T} d t \int_{\mathbb{R}^{m}}(V(y(t)+\theta(y(t), u, t), t)-V(y(t), t)) \tilde{v}(d u, d t) .
\end{aligned}
$$


COROLLARY 6.5. Let $p \triangleq \mathbf{L}(0) * \rho$. In the assumptions and notation of Theorem 6.4,

$$
\begin{aligned}
\mathbf{E} \hat{\mathbf{t}}(T)^{2}= & \int_{\mathbb{R}^{n}}|V(x, 0)|^{2} \rho(x) d x \\
& +\int_{Q}\left(\sum_{j=1}^{n}\left|\frac{\partial V}{\partial x}(x, t) \beta_{j}(x, t)\right|^{2}+\int_{\mathbb{R}^{m}} \mid V(x+\theta(x, u, t), t)\right. \\
& \left.-\left.V(x, t)\right|^{2} \Pi(d u)\right) p(x, t) d x d t .
\end{aligned}
$$

Note that if Condition 6.1(ii) is satisfied, then $\|\rho\|_{H^{0}}^{2}=\int_{\mathbb{R}^{n}} \rho(x)^{2} d x \leq\|\rho\|_{L_{\infty}\left(\mathbb{R}^{n}\right)}$, and $\rho \in H^{0}$. By definition, $p$ in Corollary 6.5 is the solution of the boundary value problem (4.2) with $\varphi=0, g=0$. Moreover, by Theorem 4.1, it follows that $p(x, t)$ is the probability density function of the process $y(t)$, and, by Corollary $4.7, p \in L_{\infty}(Q)$.

Proof OF Theorem 6.4. Set

$$
\begin{aligned}
\xi_{j}(t) & \triangleq \frac{\partial V}{\partial x}(y(t), t) \beta_{j}(y(t), t), \\
\psi(u, t) & \triangleq V(y(t)+\theta(y(t), u, t), t)-V(y(t), t) .
\end{aligned}
$$

Let $h_{\varepsilon}(x, t) \in X^{0} \cap C\left([0, T] ; C^{2}\left(\mathbb{R}^{n}\right)\right)$ be such that $\left\|h_{\varepsilon}-g_{\varepsilon}\right\|_{X^{0}} \leq \varepsilon$. Set

$$
\begin{gathered}
V_{\varepsilon} \triangleq L_{(0)}, \quad \lambda_{\varepsilon}(t) \triangleq \int_{0}^{t} h_{\varepsilon}(y(s), s) d s, \\
\xi_{j, \varepsilon}(t) \triangleq \frac{\partial V_{\varepsilon}}{\partial x}(y(t), t) \beta_{j}(y(t), t), \\
\psi_{\varepsilon}(u, t) \triangleq V_{\varepsilon}(y(t)+\theta(y(t), u, t), t)-V_{\varepsilon}(y(t), t) .
\end{gathered}
$$

By definition, we have that $h_{\varepsilon}=-\partial V_{\varepsilon} / \partial t-\mathscr{A} V_{\varepsilon}$ and $V_{\varepsilon}(x, T)=0$. By the generalized Itô formula (cf. [6, page 272]), it follows that

$$
\begin{aligned}
-V_{\varepsilon}(a, 0)= & V_{\varepsilon}(y(T), T)-V_{\varepsilon}(a, 0) \\
= & -\int_{0}^{T} h_{\varepsilon}(y(t), t) d t+\sum_{j=1}^{n} \int_{0}^{T} \xi_{j}(y(t), t) d w_{j}(t) \\
& +\int_{0}^{T} d t \int_{\mathbb{R}^{m}}\left(V_{\varepsilon}(y(t)+\theta(y(t), u, t), t)-V_{\varepsilon}(y(t), t)\right) \tilde{v}(d u, d t) .
\end{aligned}
$$

Hence

$$
\begin{aligned}
\lambda_{\varepsilon}(T)= & V_{\varepsilon}(a, 0)+\sum_{j=1}^{n} \int_{0}^{T} \frac{\partial V_{\varepsilon}}{\partial x}(y(t), t) \beta_{j}(y(t), t) d w_{j}(t) \\
& +\int_{0}^{T} d t \int_{\mathbb{R}^{m}}\left(V_{\varepsilon}(y(t)+\theta(y(t), u, t), t)-V_{\varepsilon}(y(t), t)\right) \tilde{v}(d u, d t) .
\end{aligned}
$$

By [6, Lemmas 2, 3 and Theorem 4, pages 293-296], it follows that the functions $V_{\varepsilon}$ and $\partial V_{\varepsilon}(x, t) / \partial x$ are bounded and continuous, then $\xi_{\varepsilon, j}(t) \in \mathscr{y}^{0}$ and $\psi_{\varepsilon}(u, t) \in \mathscr{L}^{0}$. 
By Theorem 4.1, $p=p(x, t) \triangleq \hat{\mathbf{L}}(0)^{*} \rho$ is the probability density function of the process $y(t)$. Let $W_{\varepsilon} \triangleq V_{\varepsilon}-V$. By Corollary 3.9, $\left\|W_{\varepsilon}\right\|_{Y^{1}} \rightarrow 0$ as $\varepsilon \rightarrow 0$. Then

$$
\begin{aligned}
& \mathbf{E}\left|V_{\varepsilon}(a, 0)-V(a, 0)\right|^{2}=\int_{\mathbb{R}^{n}}\left\|W_{\varepsilon}(x, 0)\right\|^{2} \rho(x) d x \\
& \leq\|\rho\|_{L_{\infty}\left(\mathbb{R}^{n}\right)}\left\|W_{\varepsilon}\right\|_{X^{0}} \longrightarrow 0, \\
& \mathbf{E} \int_{0}^{T}\left|\xi_{j, \varepsilon}(t)-\xi_{j}(t)\right|^{2} d t=\sum_{j=1}^{n} \mathbf{E} \int_{0}^{T}\left|\frac{\partial W_{\varepsilon}}{\partial x}(y(t), t) \beta_{j}(y(t), t)\right|^{2} d t \\
& =\int_{Q}\left|\frac{\partial W_{\varepsilon}}{\partial x}(x, t)\right|^{2} p(x, t) d x d t \\
& \leq \mathrm{const}\|p\|_{L_{\infty}(Q)}\left\|W_{\varepsilon}\right\|_{Y^{1}} \longrightarrow 0 \\
& \mathbf{E} \int_{0}^{T} d t \int_{\mathbb{R}^{n}}\left|\psi_{\varepsilon}(u, t)-\psi(u, t)\right|^{2} \Pi(d u)=\mathbf{E} \int_{0}^{T}\left|\left(\mathscr{f}(t) W_{\varepsilon}\right)(y(t), t)\right|^{2} d t \\
& =\int_{Q}\left|\left(\mathscr{g}(t) W_{\varepsilon}\right)(x, t)\right|^{2} p(x, t) d x d t
\end{aligned}
$$

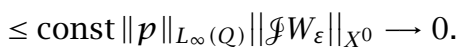

Then

$$
\begin{aligned}
\mathbf{E}\left|\hat{\mathbf{t}}(T)-\lambda_{\varepsilon}(T)\right|^{2}= & \mathbf{E}\left|V_{\varepsilon}(a, 0)-V(a, 0)\right|^{2} \\
& +\sum_{j=1}^{n} \mathbf{E} \int_{0}^{T}\left|\xi_{j, \varepsilon}(t)-\xi_{j}(t)\right|^{2} d t \\
& +\mathbf{E} \int_{0}^{T} d t \int_{\mathbb{R}^{n}}\left|\psi_{\varepsilon}(u, t)-\psi(u, t)\right|^{2} \Pi(d u) \longrightarrow 0 .
\end{aligned}
$$

Furthermore,

$$
\begin{aligned}
\mathbf{E}\left|l_{\varepsilon}(T)-\lambda_{\varepsilon}(T)\right|^{2} & =\int_{0}^{T} \int_{\mathbb{R}^{n}}\left|g_{\varepsilon}(x, t)-h_{\varepsilon}(x, t)\right|^{2} p(x, t) d t \\
& \leq\|p\|_{L_{\infty}(Q)} \mid\left\|g_{\varepsilon}-h_{\varepsilon}\right\|_{X^{0}} \longrightarrow 0 .
\end{aligned}
$$

This completes the proof of Theorem 6.4.

Proof OF Corollary 6.5. The proof can be easily obtained similar to (6.12).

\section{Equations for the characteristic function of the occupation time}

THEOREM 7.1. Assume that Conditions 3.1, 4.3, 4.6, and 6.1 are satisfied, and that $g \in \mathscr{X}$. Let $v \in \mathbb{R}$ be given, and let $z \triangleq i v$, where $i=\sqrt{-1}$. Let $V \triangleq z L(z g) g$. Then $V \in Y^{1}$, and

$$
1+(V(\cdot, 0), \rho)_{H^{0}}=\operatorname{E} \exp \{z \hat{\mathbf{t}}(T)\} .
$$


Proof. Let $h_{\varepsilon}$ be such that $h_{\varepsilon}(x, t) \in X^{0} \cap C\left([0, T] ; C^{2}\left(\mathbb{R}^{n}\right)\right)$ and $\left\|h_{\varepsilon}-g_{\varepsilon}\right\|_{\mathscr{L}} \leq \varepsilon$. Set $\lambda_{\varepsilon}(t) \triangleq \int_{0}^{t} h_{\varepsilon}(y(s), s) d s$. Let

$$
V_{\varepsilon}(x, s) \triangleq \mathbf{E}\left\{z \int_{s}^{T} h_{\varepsilon}(y(t), t) \exp \left(z \int_{s}^{t} h_{\varepsilon}(y(r), r) d r\right) d t \mid y(s)=x\right\} .
$$

It is easy to see that

$$
V_{\varepsilon}(x, s)=\mathbf{E}\left\{\exp \left(z \int_{s}^{T} h_{\varepsilon}(y(t), t) d t\right) \mid y(s)=x\right\}-1 .
$$

By [6, Theorem 1, page 301], applied after a small modification for a non-homogeneous integro-differential equation, it follows that $V_{\varepsilon}=z L\left(z h_{\varepsilon}\right) h_{\varepsilon}$, that is, $V_{\varepsilon}$ is the solution of the problem

$$
\frac{\partial V_{\varepsilon}}{\partial t}+\mathscr{A} V_{\varepsilon}+z h_{\varepsilon} V_{\varepsilon}=-z h_{\varepsilon}, \quad V_{\varepsilon}(x, T)=0
$$

By Lemma 5.2 it follows that $\left\|\mathfrak{g}-g_{\varepsilon}\right\|_{\mathscr{Q}} \rightarrow 0$ as $\varepsilon \rightarrow 0+$. Hence $\left\|g-h_{\varepsilon}\right\|_{\mathscr{X}} \rightarrow 0$ as $\varepsilon \rightarrow 0+$. By Corollary 3.9, it follows that $\left\|V-V_{\varepsilon}\right\|_{Y^{1}} \rightarrow 0$. Hence $\left(V_{\varepsilon}(\cdot, 0), \rho\right)_{H^{0}} \rightarrow(V(\cdot, 0), \rho)_{H^{0}}$. It was shown in the proof of Theorem 6.4 that $\mathbf{E}\left|\lambda_{\varepsilon}(T)-\hat{\mathbf{t}}(T)\right|^{2} \rightarrow 0$. Then $\lambda_{\varepsilon}(T)$ converges to $\hat{\mathbf{t}}(T)$ in distribution, and $\mathbf{E} e^{z \lambda_{\varepsilon}(T)} \rightarrow \mathbf{E} e^{z \hat{\mathbf{t}}(T)}$ for each $z=i v, v \in \mathbb{R}$. Then the proof follows.

ACKNOWLEDGEMENT. This work was supported by Russian Foundation for Basic Research.

\section{REFERENCES}

[1] R. F. Bass, Occupation times of d-dimensional semimartingales, Seminar on Stochastic Processes, 1982 (Evanston, Ill., 1982), Progr. Probab. Statist., vol. 5, Birkhäuser Boston, Massachusetts, 1983, pp. 51-96. MR 85j:60080. Zbl 0526.60045.

[2] A. N. Borodin, Brownian local time, Russian Math. Surveys 44 (1989), no. 2, 1-51. Zbl 0705.60064 .

[3] N. G. Dokuchaev, Local sojourn time of diffusion and degenerating processes on a mobile surface, Theory Probab. Appl. 43 (1999), no. 2, 171-188.

[4] M. G. Garroni and J.-L. Menaldi, Green Functions for Second Order Parabolic IntegroDifferential Problems, Pitman Research Notes in Mathematics Series, vol. 275, Longman Scientific \& Technical, Harlow, 1992. MR 94g:35110. Zbl 0806.45007.

[5] D. Geman and J. Horowitz, Occupation densities, Ann. Probab. 8 (1980), no. 1, 1-67. MR 81b:60076. Zbl 0499.60081.

[6] Ĭ. İ. Gīhman and A. V. Skorohod, Stochastic Differential Equations, Ergebnisse der Mathematik und ihrer Grenzgebiete, vol. 72, Springer-Verlag, New York, 1972. MR 49\#11625. Zbl 0242.60003.

[7] O. A. Ladyženskaja, V. A. Solonnikov, and N. N. Ural'ceva, Linear and Quasilinear Equations of Parabolic Type, Translations of Mathematical Monographs, vol. 23, American Mathematical Society, Rhode Island, 1967, translated from the Russian by S. Smith. MR 39\#3159b.

[8] P. McGill, Markov properties of diffusion local time: a martingale approach, Adv. in Appl. Probab. 14 (1982), no. 4, 789-810. MR 84f:60106. Zbl 0502.60062.

[9] P. A. Meyer, Un cours sur les intégrales stochastiques, Séminaire de Probabilités, X (Seconde partie: Théorie des intégrales stochastiques, Univ. Strasbourg, Strasbourg, année universitaire 1974/1975), Lecture Notes in Math., vol. 511, Springer, Berlin, 1976, pp. 245-400 (French). MR 58\#18721. Zbl 0374.60070. 
[10] J. P. Nolan, Local nondeterminism and local times for stable processes, Probab. Theory Related Fields 82 (1989), no. 3, 387-410. MR 91b:60060. Zbl 0687.60071.

[11] P. Protter, Stochastic Integration and Differential Equations. A New Approach, Applications of Mathematics, vol. 21, Springer-Verlag, Berlin, 1990. MR 91i:60148. Zbl 0694.60047.

[12] J. Rosen and M. Yor, Tanaka formulae and renormalization for triple intersections of Brownian motion in the plane, Ann. Probab. 19 (1991), no. 1, 142-159. MR 92c:60108. Zbl 0719.60084 .

[13] P. Salminen, On the distribution of supremum of diffusion local time, Statist. Probab. Lett. 18 (1993), no. 3, 219-225. MR 94h:60115. Zbl 0796.60080.

N. G. Dokuchaev: The Institute of Mathematics and Mechanics, St. Petersburg State UNIVERSITY, 198904, RUSSIA

E-mail address: dokuchaev@pobox.spbu.ru 




Advances in

Operations Research



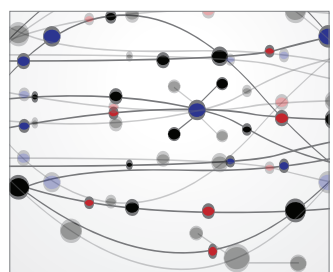

\section{The Scientific} World Journal
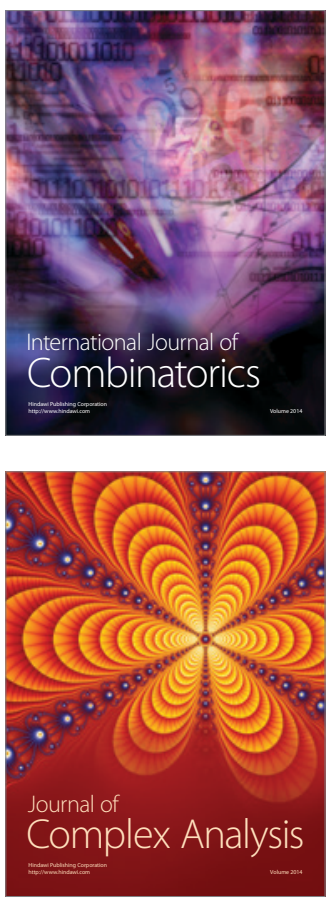

International Journal of

Mathematics and

Mathematical

Sciences
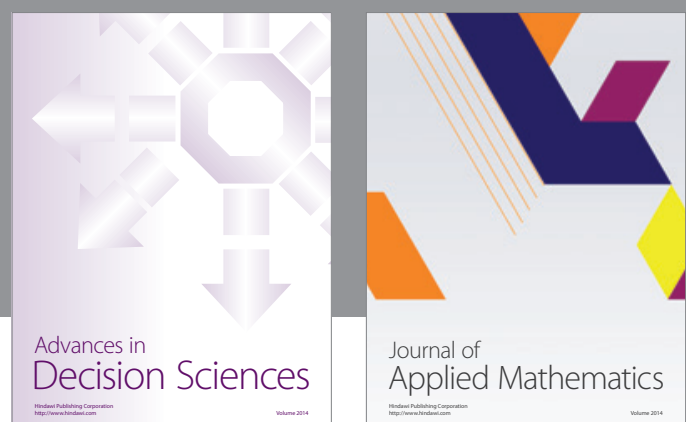

Journal of

Applied Mathematics
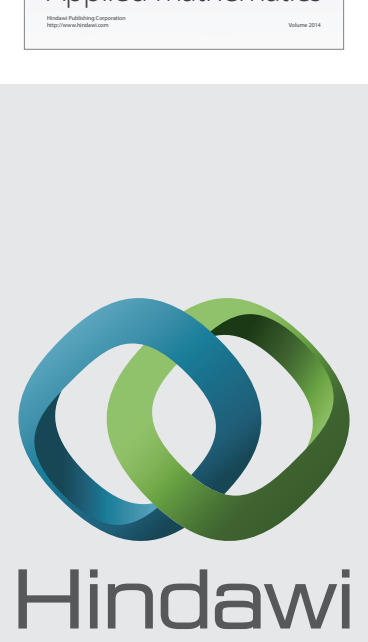

Submit your manuscripts at http://www.hindawi.com
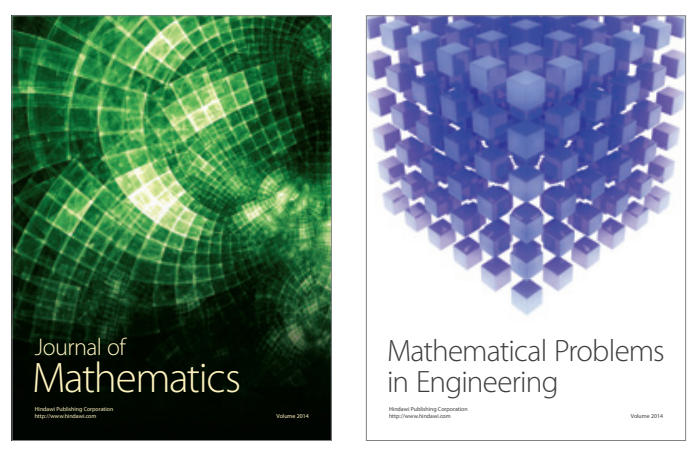

Mathematical Problems in Engineering


Journal of

Function Spaces
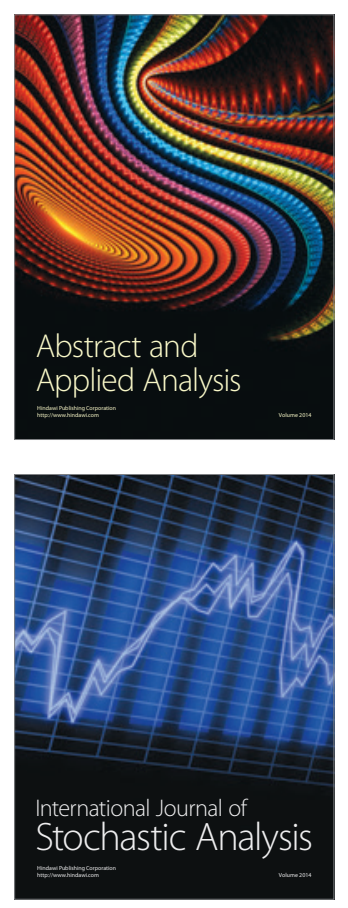

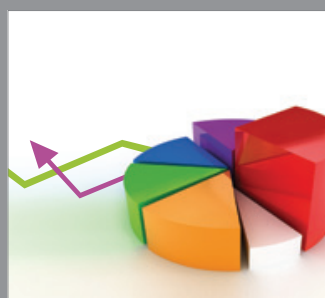

ournal of

Probability and Statistics

Promensencen
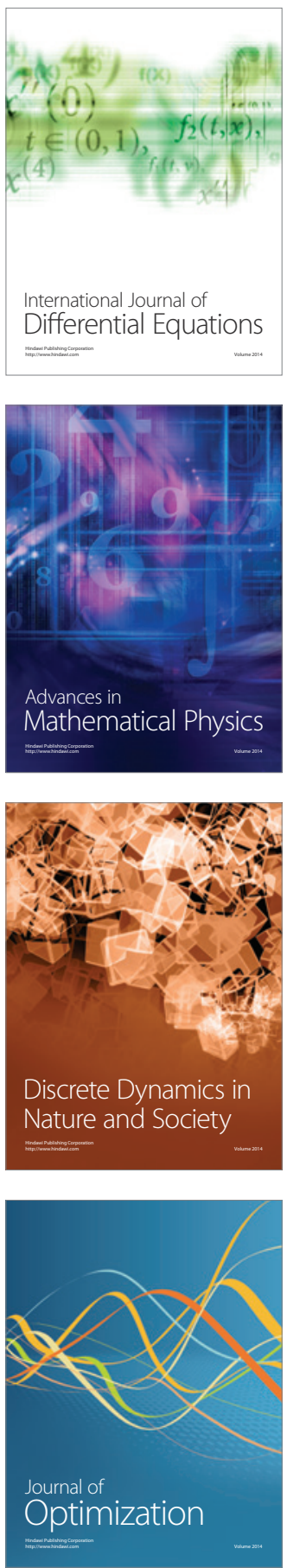